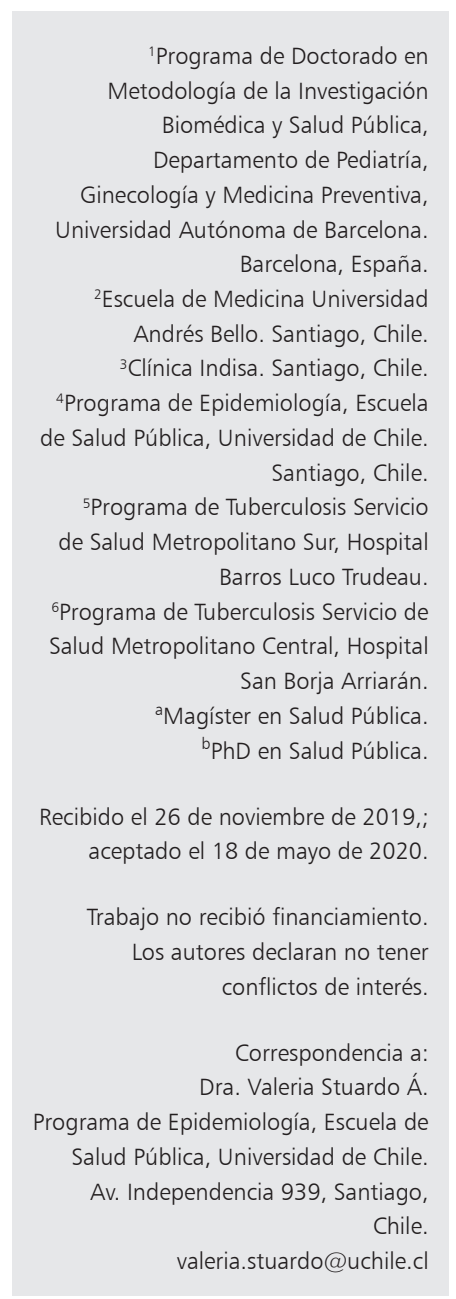

\section{Evolución de la coinfección por TB/VIH en la Región Metropolitana de Chile, 2005 a 2018}

\author{
CLAUDIO OLMOS ${ }^{1,2,3, a}$, VALERIA STUARDO ${ }^{4, b}$, \\ PAULINA RAMONDA ${ }^{5}$, CARLOS PEÑA ${ }^{6}$
}

\section{Evolution of tuberculosis/HIV coinfection in Metropolitan Santiago, Chile from 2005 to 2018}

\begin{abstract}
Background: Tuberculosis (TB)/HIV coinfection has a high mortality rate. Aim: To describe socio-epidemiological characteristics of tuberculosis (TB)-HIV coinfection, and aspects associated with its prevalence, from 2005 to 2018. Material and Methods: Analysis of the registry of the tuberculosis control and elimination program of three public health services of metropolitan Santiago. The variables considered were: TB/HIV coinfection, age, sex, location of tuberculosis, bacteriological confirmation, incarceration, commune of residence, country of origin and effectiveness of the tuberculosis therapy. Results: We analyzed 7507 TB cases, of whom $12 \%$ corresponded to cases of coinfection. The number of coinfections doubled in the last 6 years. In 2018, 45\% of coinfection cases occurred in migrants. Of the total cases evaluated, $53 \%$ were successfully treated and $28 \%$ died. Conclusions: The remarkable increase in TB/HIV coinfection, urgently demands new prevention and control strategies, aimed at the most vulnerable groups.
\end{abstract}

(Rev Med Chile 2020; 148: 618-625)

Key words: Epidemiological Monitoring; HIV; Tuberculosis.

\section{L} a tuberculosis (TB) y la infección por virus de inmunodeficiencia humana adquirida (VIH) continúan siendo epidemias altamente letales a nivel mundial. El 2017 hubo aproximadamente 10 millones de casos nuevos de TB y cerca de 1,3 millones de muertes por esta causa $^{1}$, mientras que ese mismo año, se produjeron 1,8 millones de nuevas infecciones por el VIH y fallecieron 940.000 personas en todo el mundo, a causa de enfermedades relacionadas con el sida².

La coinfección por ambos agentes, resulta aún más letal, ya que se potencian uno a otro, apresurando el deterioro de las funciones inmunológicas, pudiendo llevar a una muerte prematura si no se trata adecuada y oportunamente ${ }^{3}$.

La evidencia establece que el riesgo que tiene una persona con VIH de desarrollar una TB activa, es cerca de 20 veces mayor que el riesgo que corre el resto de la población mundial ${ }^{1}$.

De hecho, la TB sigue siendo la principal causa de muerte por enfermedad infecciosa en personas con VIH $(31,9 \%)^{2,4}$. Un estudio determinó mediante autopsias de pacientes con VIH, que en $66,7 \%$ de ellos se detectó infección por $\mathrm{Myco-}$ bacterium tuberculosis ${ }^{5}$. Asimismo, se estima que aproximadamente, una de cada cinco muertes por TB correspondería a personas seropositiva para el VIH $(18,8 \%)^{1}$.

El problema se agudiza, si se estima que 49\% de las personas que viven con TB y VIH desconocen su coinfección ${ }^{6}$, y solo $39 \%$, ha recibido tratamiento para ambas infecciones ${ }^{7}$. Dificultades 
como la capacidad de identificar oportunamente los casos, implementar un sistema de notificación eficiente y la falta de identificación de grupos de riesgo, pueden llevar a un subdiagnóstico tanto de $\mathrm{VIH}$, como de la coinfección ${ }^{8}$.

Así, son muchos los factores que inciden en el riesgo de las personas, pero las limitaciones y condiciones de vida que deben enfrentar los migrantes, los sitúa a ellos como un grupo altamente vulnerable ${ }^{9}$.

La migración y las complejas condiciones de vida de los migrantes en los países de destino pueden aumentar la vulnerabilidad de las personas y por ende aumentan su riesgo de adquirir enfermedades infecciosas ${ }^{10-12}$.

Estudios en Europa han mostrado que los migrantes diagnosticados con $\mathrm{VIH}$, tienden a consultar tardíamente por atención y tratamiento, en comparación a los nacionales ${ }^{13}$. Adicionalmente, la evidencia muestra que la población migrante en Europa con coinfección por TB/VIH, han sido especialmente propensos al fracaso del tratamiento, a la resistencia farmacológica y a una muerte prematura $^{14}$.

Por todo aquello, es que la coinfección por TB/VIH y la identificación de grupos vulnerables, suponen un importante desafío, tanto para el diagnóstico como para el control de la enfermedad. Así, el propósito de este estudio es describir la evolución socio-epidemiológica de la coinfección por TB/VIH, y los aspectos asociados a la ocurrencia de casos, desde 2005 a 2018 en los principales servicios de salud de la Región Metropolitana de Chile.

\section{Materiales y Métodos}

Se trata de un estudio descriptivo con componente ecológico, en el cual se analizaron los casos de coinfección por TB/VIH registrados por el Programa de Control y Eliminación de tuberculosis (PROCET) en los Servicios de Salud Metropolitano Central (SSMC), Norte (SSMN) y Sur (SSMS), entre los años 2005 a 2018.

Para el SSMC y SSMS, se contó con la totalidad de los registros desde 2005 a 2018, mientras que, para el SSMN, sólo se dispuso de registros a partir de 2012. La población cubierta por estos servicios fue de 3.429 .872 personas en 2018 , lo que representa $48,2 \%$ de todos los habitantes de la Región Metropolitana (RM) y 19,5\% de la población total del país.
Informes preliminares muestran que estos tres Servicios tienen altas tasas de TB y reciben una importante cantidad de extranjeros. En efecto, $68 \%$ de los migrantes de la RM se concentra en este territorio, representando aproximadamente $9,8 \%$ del total de la población cubierta por los tres Servicios de Salud.

Se revisaron los casos diagnosticados y se recogieron los siguientes antecedentes provenientes del formulario de notificación obligatoria: coinfección por TB/VIH, sexo, edad, localización de la tuberculosis (pulmonar y extrapulmonar), confirmación bacteriológica (frotis, cultivo, reacción en cadena de la polimerasa (PCR), y mediante otros hallazgos), privación de libertad, comuna de residencia, país de procedencia y efectividad de la terapia antituberculosis.

Para la variable efectividad de la terapia antituberculosis se definieron 4 atributos de acuerdo a los criterios que describe la OMS: "éxito global" del tratamiento si es que el paciente culmina su terapia; "abandono o pérdida de seguimiento" si registra inasistencia continua a la terapia por más de 30 días; "fracaso", cuando la baciloscopía persiste positiva hasta el cuarto mes de tratamiento, o si luego de hacerse negativa reaparece positiva durante la terapia y "muerte", si es que esta ocurre durante el tratamiento, independiente de la causa.

Se utilizó el paquete estadístico STATA 13.0 y el software Microsoft Excel 2016 para el análisis de datos. Se realizó un análisis de correlación lineal de Pearson entre los casos de coinfección TB/VIH y el porcentaje de casos extranjeros. Se usaron pruebas de Chi-cuadrado para analizar los resultados de la terapia. Se consideró estadísticamente significativo a un $\mathrm{p}$ valor menor a 0,05 .

Se contó con la autorización de los encargados del PROCET de los Servicios de Salud Metropolitano Central, Norte y Sur, así como la aprobación del Comité de Bioética del SSMC.

\section{Resultados}

Durante el periodo estudiado, en los tres servicios de salud, se registraron 7.507 casos de TB. De ellos, 906 correspondieron a casos de coinfección por $\mathrm{TB} / \mathrm{VIH}$, lo que representa $12,1 \%$ (Mín = 7\% en el año 2007; Máx $=15,6 \%$ en el año 2015), mostrando una leve tendencia al alza a lo largo del periodo. El 86,9\% de los casos de coinfección 
fueron de sexo masculino. Mientras que la edad promedio de casos de TB sin VIH fue de 44,5 años, la edad promedio en los casos de coinfección fue de 38 años; casi 7 años más joven.

Del total de casos de coinfección, 72,1\% correspondió a tuberculosis pulmonar (TBP) siendo confirmados bacteriológicamente 79,8\% de ellos. Por otro lado, 27,9\% de los casos se describieron como TB extrapulmonar, destacándose las formas ganglionar y pleural como las más frecuentes.

El 2,0\% de los casos ocurrió en personas privadas de libertad, los que provinieron mayoritariamente del SSMC (67,1\% de los casos), el cual da cobertura al recinto penitenciario más grande del país. En la Tabla 1 se observan las características demográficas y clínicas de los casos en personas con y sin coinfección por VIH.

En cuanto a la letalidad, se evidencia que las personas con coinfección por TB/VIH presentan un mayor riesgo de morir. Mientras que la letalidad por TB en personas sin VIH fue de 11,6\% en promedio anual (Mín. = 7,6\%; Máx. $=19,6 \%$; $\mathrm{DE}=3,2 \%)$, la letalidad por coinfección fue de 29,7\% (Mín. = 17,1\%; Máx. $=46,4 \% ; \mathrm{DE}=9,15$ ). La evolución de la letalidad en personas con y sin coinfección, se muestra en la Figura 1.

En cuanto a la incidencia de casos, se aprecia un importante aumento en el número de casos de coinfección en los últimos 4 años (media $=96$ casos por año), cercano a duplicar el promedio de casos observados entre el 2005 y 2014 (media $=52,3$ casos por año), aunque la proporción respecto del total de casos de TB se mantuvo relativamente constante, con un crecimiento anual de $0,26 \%$.

Los casos de coinfección se concentran prin-
Tabla 1. Características sociodemográficas y clínicas de los casos de tuberculosis, en personas con VIH(+) y VIH(-), entre los años 2005 y 2018, en tres Servicios de Salud de la Región Metropolitana de Chile

\begin{tabular}{|c|c|c|}
\hline \multirow{2}{*}{$\begin{array}{l}\text { Característica socio- } \\
\text { demográfica y clínica }\end{array}$} & \multicolumn{2}{|c|}{ Casos de tuberculosis } \\
\hline & VIH (-) & VIH (+) \\
\hline \multicolumn{3}{|l|}{ Sexo (\%) } \\
\hline Femenino & 36,9 & 13,1 \\
\hline Masculino & 63,1 & 86,9 \\
\hline \multicolumn{3}{|l|}{ Edad (media en años) } \\
\hline Femenino & 47,1 & 39,7 \\
\hline Masculino & 44,3 & 37,8 \\
\hline Total & 45,4 & 38,0 \\
\hline \multicolumn{3}{|l|}{ Localización (\%) } \\
\hline Pulmonar & 75,5 & 72,1 \\
\hline Pulmonar BD* & 66,5 & 57,6 \\
\hline Extrapulmonar & 24,5 & 27,9 \\
\hline Privación de libertad (\%) & 7,7 & 2,0 \\
\hline
\end{tabular}

*Pulmonar BD: Tuberculosis pulmonar bacteriológicamente demostrada. Fuente: Elaboración propia de los autores.

cipalmente en la comuna de Santiago $(43,5 \%)$, seguido de Estación Central, Independencia, Recoleta y Pedro Aguirre Cerda $(27,4 \%$ en su conjunto), que coinciden como las comunas de mayor tasa de TB y mayor cantidad de migrantes.

Por otra parte, se observa una variación importante en el porcentaje de casos de TB/VIH en personas migrantes, quienes prácticamente no tenían presencia en las notificaciones hasta el 2012, pero exhiben un alza sostenida en los últimos años,

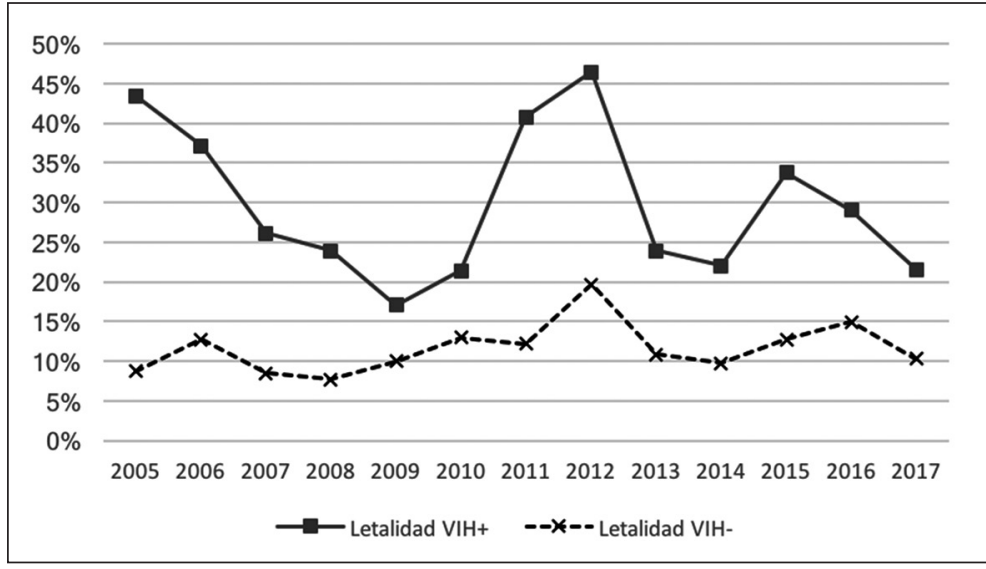

Figura 1. Letalidad por TB en personas con VIH (+) y VIH (-), en tres Servicios de Salud de la Región Metropolitana de Chile, 2005-2017. Fuente: Creación propia de los autores. 


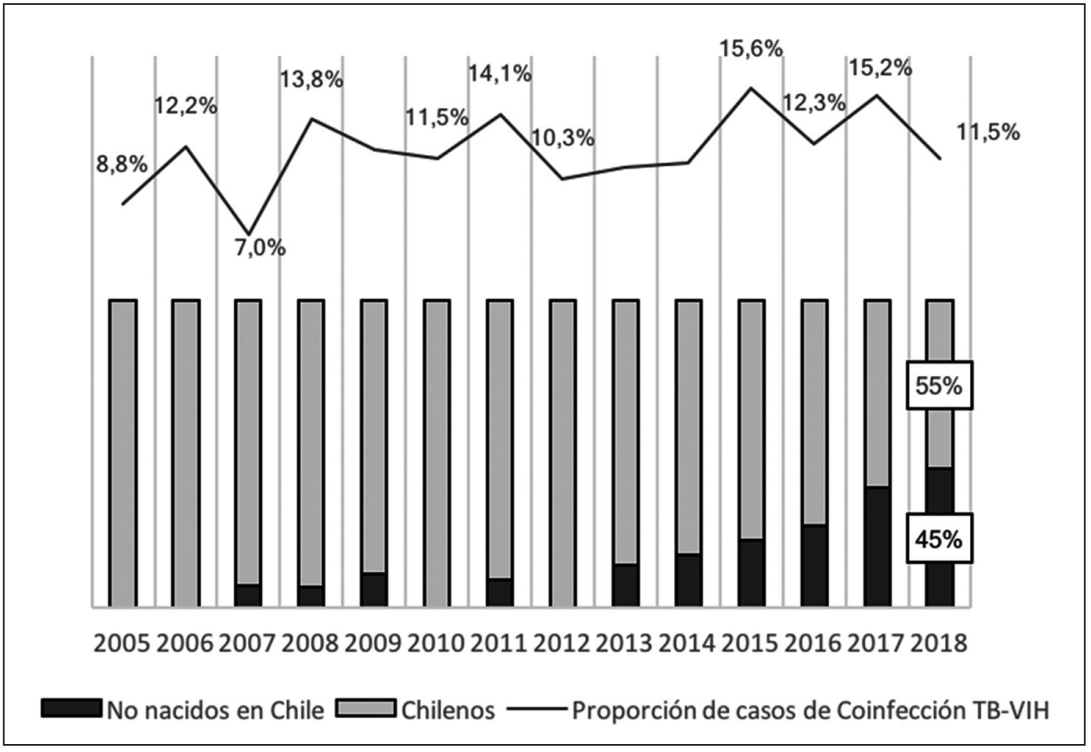

Figura 2. Proporción de casos de coinfección por TB/ $\mathrm{VIH}$ notificados al PROCET y su distribución en población chilena y extranjera, en tres Servicios de Salud de la Región Metropolitana de Chile, 20052018. Fuente: Creación propia de los autores. Al aplicarse un análisis de correlación simple, no se observa asociación entre el aumento en la proporción de casos de TB en personas migrantes y la evolución de casos de coinfección ( $r=0,38$; $p=0,17)$. llegando a constituir $45 \%$ de los casos de coinfección en el año 2018. De ellos, 42,2\% provino de Haití, 22,2\% de Perú, 11,1\% de Colombia y 20\% de países del resto de América latina.

Al aplicarse un análisis de correlación simple, no se observa asociación entre el aumento en la proporción de casos de TB en personas migrantes y la evolución de casos de coinfección $(r=0,38$; $\mathrm{p}=0,17)$. La proporción de casos de coinfección por TB/VIH, así como su distribución en población chilena y extranjera se detallan en la Figura 2.

En relación a los resultados de la terapia, solo fueron considerados 4.394 casos de TB, excluyendo aquellos casos con registros incompletos, y los casos ocurridos durante el 2018, muchos de los cuales aún no completaban su tratamiento al iniciar esta investigación. Del total de casos de coinfección por TB/VIH evaluados, se observó 53,3\% de éxito, $28,2 \%$ de muerte y $18,5 \%$ de abandono.

$\mathrm{Al}$ comparar los resultados entre personas con y sin coinfección, se aprecia que el éxito del tratamiento en personas coinfectadas es casi $20 \%$ menor que en personas sin VIH. Del mismo modo, los coinfectados exhiben una proporción de casos fallecidos el doble más alta que los seronegativos. Mediante la prueba de chi-cuadrado, se observó que estas diferencias resultaron ser estadísticamente significativas $(\mathrm{p}<0,001)$. Los resultados de la terapia anti-tuberculosis, tanto en personas coinfectadas como en personas sin VIH, se muestran en las Figuras 3 y 4.

\section{Discusión}

La población de estudio en esta investigación, abarca casi $20 \%$ de la población chilena, concentra $25,9 \%$ de los casos de TB a nivel nacional y $38,0 \%$ de los casos de coinfección por TB/VIH notificados en todo el país durante los últimos 5 años ${ }^{15}$.

Los resultados de este estudio muestran que el perfil socio-epidemiológico de las personas coinfectadas por TB/VIH ha cambiado en los últimos 14 años. Al 2018, la gran mayoría está compuesta por hombres menores de 40 años y casi la mitad de ellos pertenecen a población extranjera.

Las cifras de casos de coinfección en personas privadas de libertad, resultaron ser notoriamente más bajas que el porcentaje de casos de TB diagnosticados en población general $(2,0 \%$ y $7,7 \%$ respectivamente), aun cuando las tasas de TB como las tasas de VIH suelen ser más altas en este grupo de personas ${ }^{16-18}$. Esto podría deberse al eficiente control que ha realizado el PROCET, al insertar dentro de los recintos penales, unidades especializadas en la detección y control de la tuberculosis.

También, se ha observado un aumento de casos de coinfección en la población migrante. 

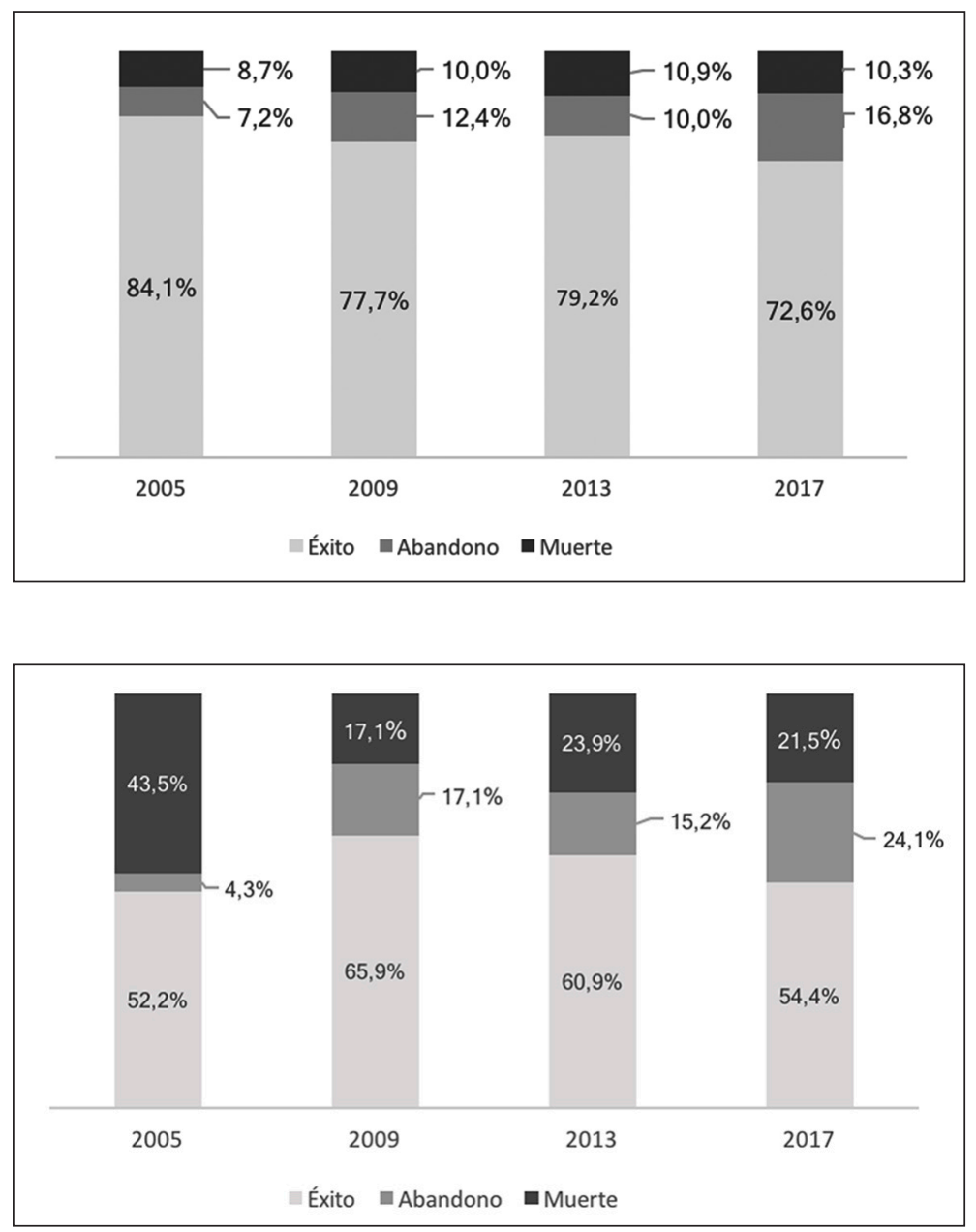

Figura 3. Resultados de la terapia en personas VIH (-), en tres Servicios de Salud de la Región Metropolitana de Chile, durante los años 2005, 2009, 2013 y 2017. Fuente: Creación propia de los autores.
Figura 4. Resultados de la terapia en personas $\mathrm{VIH}(+)$, en tres Servicios de Salud de la Región Metropolitana de Chile, durante los años 2005, 2009, 2013 y 2017. Fuente: Creación propia de los autores.
La proporción de casos de TB/VIH en extranjeros pasó de $13,8 \%$ en 2013, a 45,0\% en 2018. Como ya se dijo, este grupo constituye un foco de interés epidemiológico debido a su situación de vulnerabilidad ${ }^{12,19}$. Las situaciones que enfrentan, los exponen a una serie de factores de riesgo para ITS: violencia, abuso sexual, discriminación, deficiencias nutricionales, exposición a enfermedades infectocontagiosas, consumo de alcohol y drogas, barreras idiomáticas, aumento de relaciones sexuales sin protección, limitado o nulo acceso a servicios de salud ${ }^{19,20}$.

En relación con el VIH, datos epidemiológicos muestran que la proporción de personas notificadas nacidas fuera del país ha pasado de $2 \%$ a $11,6 \%$ en los últimos 20 años, siendo $85 \%$ de sexo mas- culino. El año 2017 el porcentaje de extranjeros notificados representó $19 \%$ del total de casos $^{21}$.

Por otra parte, los factores sociales antes mencionados, sumados al temor de los grupos vulnerables a una posible estigmatización, serían motivos para que, personas en situación de vulnerabilidad, evite consultar, provocando un posible subdiagnóstico tanto de TB como de $\mathrm{VIH}^{8,22}$. Según el reporte oficial de 2018, a nivel mundial, solo en $13 \%$ de todos los casos de TB se confirmó la coinfección por VIH, lo que representaría sólo $46 \%$ del número de casos esperados ${ }^{22}$.

En Chile, la TB venía declinando progresivamente desde el siglo pasado, sin embargo, desde 2014 a la fecha se ha descrito una etapa de reemergencia epidemiológica. Entre los grupos de 
vulnerabilidad con mayor aumento porcentual entre los casos, se encuentran los extranjeros, y en menor grado, los pacientes con $\mathrm{VIH} / \mathrm{sida}^{23}$.

Según el último informe de situación de tuberculosis, la proporción de coinfección a nivel nacional, alcanzó $9,6 \%$ de todos los casos de $\mathrm{TB}$, aumentando en $10 \%$, respecto de lo que había ocurrido el año anterior ${ }^{15}$ cifra que podría continuar en ascenso durante los próximos años. Aquella suposición está basada en tres fenómenos socio-epidemiológicos que afectan a la región: a) Chile fue el país que reportó el mayor aumento de casos de VIH en los últimos cinco años en América latina $^{7}$; en efecto, la Tasa de Incidencia de VIH en Chile aumentó casi en 200\% pasando de 17,5 x 100.000 en el 2010 a 37,5 x 100.000 en $2018^{24}$; b) Las tasas de incidencia de TB en el país han exhibido un sostenido aumento desde el 2012, en un retroceso sanitario nunca antes visto; el último informe de situación cifra este indicador en $14,7 \times 100.000$, siendo la más alta de los últimos 15 años ${ }^{15}$; c) Aumento de la población migrante en el país, que según cifras oficiales habría aumentado en $240 \%$ entre el 2012 y $2016^{25,26}$.

Todo aquello, hace aún más preocupante los actuales resultados de la terapia anti-tuberculosis; en la cohorte de pacientes del 2017, se observa que $24,1 \%$ abandonó la terapia y $21,5 \%$ falleció. En efecto, la coinfección TB/VIH implica per se, un riesgo de letalidad 3 veces mayor que la TB por sí misma ${ }^{27}$, por lo tanto, se requiere de mejores métodos de pesquisa y estudio de contacto más eficientes ${ }^{1,28}$, como por ejemplo la implementación de agentes comunitarios en salud ${ }^{29}$.

En términos de VIH, es urgente implementar la vigilancia de segunda generación como política pública, es decir, un sistema que vaya más allá del análisis de situación tradicional y permita analizar más a fondo aspectos sociales, económicos y culturales de la transmisión de la enfermedad. Asimismo, se debe fortalecer el testeo de base comunitaria lo que permitiría no sólo acceder a las poblaciones más vulnerables, sino también, identificar estrategias preventivas efectivas y la rápida vinculación de las personas al sistema sanitario ${ }^{30}$. Lo anterior, sería aún más efectivo si se pudiese implementar universalmente los test diagnósticos en el mismo lugar donde la persona se atiende (point of care testing), mediante los que se podría detectar oportunamente las manifestaciones de TB en personas con $\mathrm{VIH}^{31}$.
Algunas limitaciones de este estudio fueron la existencia de algunos registros incompletos, como es el caso de los resultados de la terapia y el hecho de que solo se contó con registros del SSMN a partir del 2012. A pesar de ello, este trabajo consiguió estudiar casi $40 \%$ de los casos de coinfección por TB/VIH ocurridos durante los últimos años, en todo el país, lo que constituye una gran fortaleza.

En conclusión, los resultados de este estudio muestran un notable aumento de casos de coinfección por TB/VIH, lo que, sumado a un aumento en las tasas de incidencia tanto de TB, como de VIH en el país, supone un escenario adverso que demanda urgentemente la implementación de nuevas estrategias de prevención y control, en todos los grupos de mayor vulnerabilidad.

\section{Referencias}

1. World Health Organization. Global 2018 TB report. Vol. 69, WHO report. 2018. 683-90 p. [ISBN 978-92-4156564-6]

2. Joint United Nations, Programme on HIV/AIDS [Internet]. Suiza: UNAIDS c2018 [citado el 29 de marzo de 2020]. Available from: http://www.unaids.org/es

3. Bruchfeld J, Correia-Neves M, Källenius G. Tuberculosis and HIV Coinfection. Cold Spring Harb Perspect Med 2015; 5 (7): a017871. [doi: 10.1101/cshperspect. a017871].

4. Estrategia ONUSIDA 2016-2021. Acción acelerada para acabar con el Sida. [Internet]. Suiza: ONUSIDA c2016 [citado el 9 de marzo de 2020]. Available from: https://www.unaids.org/sites/default/files/media_asset/ UNAIDS-strategy-2016-2021_es.pdf.

5. Wong E, Omar T, Setlhako G, Osih R, Feldman C, Murdoch D, Martinson N, Bangsberg D, Venter W. Causes of Death on Antiretroviral Therapy: A Post-Mortem Study from South Africa. PLoS One 2012; 7 (10): e47542. [doi.org/10.1371/journal.pone.0047542].

6. UNAIDS. Fact sheet-Worl Aids day 2018. [Internet]. Suiza: UNAIDS c2018 [cited 2020 Mar 09]. Available from: http://www.unaids.org/sites/default/files/media_asset/UNAIDS_FactSheet_en.pdf.

7. Joint United Nations Programme on HIV/AIDS. Ending Aids Progress Towards the 90-90-90 Targets. Glob Aids Updat. 2017 [Internet] Suiza: UNAIDS c2017 [citado el 9 de marzo de 2020]. Available from: https://www. unaids.org/sites/default/files/media_asset/Global_AIDS_ update_2017_en.pdf.

8. Reyes-Urueña J, Campbell C, Vives N, Esteve A, Am- 
brosioni J, Tural C, Ferrer E, Casabona; et PISCIS investigators. Estimating the HIV undiagnosed population in Catalonia, Spain: descriptive and comparative data analysis to identify differences in MSM stratified by migrant and Spanish-born population. BMJ Open 2018; 8 (2): e018533. [doi: 10.1136/bmjopen-2017-018533].

9. Pareek M, Greenaway C, Noori T, Munoz J, Zenner D, Toms C, et al. The impact of migration on tuberculosis epidemiology and control in high-income countries: a review. BMC Med 2016; 14 (1): 48. [doi: 10.1186/ s12916-016-0595-5].

10. Olawore O, Tobian A, Kagaayi J, Bazaale J, Nantume B, Grabowski M, et al. Migration and risk of HIV acquisition in Rakai, Uganda: a population-based cohort study. Lancet HIV 2018; 5 (4): e181-9. [doi: 10.1016/ S2352-3018(18)30009-2].

11. Tomás BA, Pell C, Cavanillas AB, Solvas JG, Pool R, Roura M. Tuberculosis in migrant populations. A systematic review of the qualitative literature. PLoS One. 2013; 8 (12): 1-12. [doi: 10.1371/journal.pone.0082440].

12. Hayward S, Harding RM, McShane H, Tanner R. Factors influencing the higher incidence of tuberculosis among migrants and ethnic minorities in the UK. F1000. Research 2018; 7 (0): 461. [doi: 10.12688/ f1000research.14476.1].

13. Hernando V, Alvárez-del Arco D, Alejos B, Monge S, Amato-Gauci A, Noori T, et al. HIV Infection in Migrant Populations in the European Union and European Economic Area in 2007-2012. JAIDS J Acquir Immune Defic Syndr 2015; 70 (2): 204-11. [doi: 10.1097/ QAI.0000000000000717].

14. Tavares AM, Fronteira I, Couto I, Machado D, Viveiros $\mathrm{M}$, Abecasis AB, et al. HIV and tuberculosis co-infection among migrants in Europe: A systematic review on the prevalence, incidence and mortality. PLoS One 2017; 12 (9): e0185526. [doi:10.1371/journal.pone.0185526].

15. Informe de situación epidemiológica y operacional del Programa Nacional de Tuberculosis 2017. [Internet] Chile: Ministerio de Salud de Chile; c2018 [citado el 9 de marzo de 2020]. Available from: https://diprece.minsal. cl/wp-content/uploads/2018/10/2018.10.05_TUBERCULOSIS-2017.pdf.

16. Dianatinasab M, Joulaei H, Ghorbani M, Zarei N, Rezaeian S, Fararouei M, et al. Prevalence of tuberculosis in HIV-positive prisoners: A systematic review and meta-analysis. AIDS Rev 2018; 20 (2): 114-24. [doi: 10.24875/aidsrev.m18000023].

17. Guía para el control de libertad de América en poblaciones privadas de la tuberculosis Latina y el Caribe. [Internet] Estados Unidos: Organización Panamericana de la Salud; c2008 [citado el 29 de marzo de 2020].
Available from: https://www.paho.org/spanish/ad/dpc/ cd/tb-prisiones-guia-ctl.pdf.

18. Molaeipoor L, Poorolajal J, Mohraz M, Esmailnasab N. Predictors of Tuberculosis and Human Immunodeficiency Virus Co-infection: A Case-Control Study. Epidemiol Health 2014; e2014024. [doi: 10.4178/epih/ e2014024].

19. Cabieses B, Gálvez P, Ajraz N. Migración internacional y salud: el aporte de las teorías sociales migratorias a las decisiones en salud pública. Rev Peru Med Exp Salud Publica. 2018; 35 (2): 285. [doi: 10.17843/rpmesp.2018.352.3102].

20. Caro-Murillo AM, Castilla Catalán J, del Amo Valero J. Estimaciones sobre la inmigración y el VIH en España. Gac Sanit 2010; 24 (6): 502-3. [doi:10.1016/j.gaceta.2010.07.006].

21. Boletín Vigilancia de laboratorio: Resultados confirmación de infección por VIH en Chile, 2010-2015. [Internet] Chile: Instituto de Salud Pública; c2016 [citado el 9 de marzo de 2020]. Available from: http://www.ispch. cl/sites/default/files/BoletinVIH-15112017A.pdf.

22. Rocha-Jiménez T, Morales-Miranda S, Fernández-Casanueva C, Brouwer KC, Goldenberg SM. Stigma and unmet sexual and reproductive health needs among international migrant sex workers at the Mexico-Guatemala border. Int J Gynecol Obstet 2018; 143 (1): 37-43. [doi: 10.1002/ijgo.12441].

23. Fica A, Herrera T, Aguilera X. El deterioro de la tuberculosis en Chile. Rev Med Chile 2019; 147: 1042-52. [doi: 10.4067/S0034-98872019000801042].

24. Vigilancia de laboratorios [Internet]. Chile: Instituto de Salud Pública; c2019 [citado el 9 de marzo de 2020]. Available from: http://www.ispch.cl/biomedicos/subdepto_enf_virales/sida/vigilancia.

25. Migración en Chile 2005-2014. [Internet]. Chile: Departamento de Extranjería y Migración del Ministerio del Interior y Seguridad Pública. c2014 [citado el 9 de marzo de 2020]. Available from: https://www.extranjeria.gob.cl/media/2019/04/Anuario.pdf

26. Anuario Estadístico: Estadísticas Migratorias 2015. [Internet]. Chile: Departamento de Extranjería y Migración del Ministerio del Interior y Seguridad Pública. c2017 [citado el 9 de marzo de 2020]. Available from: https:// www.extranjeria.gob.cl/media/2019/04/AnuarioEstadisticoNacionalDEM2015.pdf.

27. Situación de control de la tuberculósis en las Américas. [Internet] Estados Unidos: Organización Panamericana de la Salud; c2018 [citado el 9 de marzo de 2020]. Available from: https://www.paho.org/hq/index. php?option $=$ com_docman\&view $=$ download\&category_slug=presentaciones-5882\&alias=44088-dia-mun- 
dial-tuberculosis-2018-situacion-control-tb-americas-088\&Itemid=270\&lang=es.

28. Viana PV de S, Redner P, Ramos JP. Fatores associados ao abandono e ao óbito de casos de tuberculose drogarresistente (TBDR) atendidos em um centro de referência no Rio de Janeiro, Brasil. Cad Saude Publica. 2018; 34 (5). [doi:10.1590/0102-311x00048217].

29. Muñoz MP, Orcau A, Caylà J. Tuberculosis En Barcelo- na. Rev Esp Salud Pública 2009; 83: 751-7 [doi:10.4321/ S1135-57272014000600011].

30. Stuardo V. La prevención olvidada: reemergencia del VIH en Chile. Rev Chil Infectol. 2017; 34 (4): 419-20. [doi: 10.4067/s0716-10182017000400419].

31. Arias F, Herrera T. Nuevos métodos para el diagnóstico de la tuberculosis. Rev Chil Enferm Respir 2016; 32: 254-9 [doi:10.4067/S0717-73482016000400007]. 